\title{
Research on the application of active sound barriers for the transformer noise abatement
}

\author{
Sheng $\mathrm{Hu}^{1, \mathrm{a}}$, Shao Yi Chen ${ }^{1}$, Hai Shan Zou ${ }^{2}$ and Tie Nan $\mathrm{Li}^{1}$ \\ ${ }^{1}$ State Grid Hunan Electric Power Corporation Research Institute, 410007Changsha, Hunan Province, China \\ ${ }^{2}$ Key Laboratory of Modern Acoustics, Institute of Acoustics, Nanjing University, 210093Nanjing, Jiangsu Province, China
}

\begin{abstract}
Sound barriers are a type of measure most commonly used in the noise abatement of transformers. In the noise abatement project of substations, the design of sound barriers is restrained by the portal frames which are used to hold up outgoing lines from the main transformers, which impacts the noise reduction effect. If active sound barriers are utilized in these places, the noise diffraction of sound barriers can be effectively reduced. At a $110 \mathrm{kV}$ Substation, an experiment using a 15-channel active sound barrier has been carried out. The result of the experiment shows that the mean noise reduction value (MNRV) of the noise measuring points at the substation boundary are 1.5 $\mathrm{dB}$ (A). The effect of the active noise control system is impacted by the layout of the active noise control system, the acoustic environment on site and the spectral characteristic of the target area.
\end{abstract}

\section{Introduction}

Back in 1956, W.B.Convor from General Electric Company tried to utilize the active sound attenuation technology in controlling transformer noise. As the sound field of transformers is a free sound field in three dimensions, the fulfilment of the task back then, under premature conditions, was much too difficult, whether in sound field analysis or system implementation; therefore, the plan was abandoned, and was replaced by the passive noise control method ${ }^{[1]}$. In 1980, the American Angevine Company successfully developed an active sound attenuation method for transformers, and conditions for the commercial application of active sound attenuation became mature gradually since 1996 . The electric power sector of the United States has installed active sound attenuation system for 10 transformers, and countries such as Germany, Britain and Japan have adopted the active sound attenuation method to reduce the running noises of transformers, which turned out to be obviously effective $e^{[2 \sim 4]}$. Sound barriers are a type of measure most commonly used in the noise abatement of transformers. In the noise abatement project of substations, the design of sound barriers is restrained by the portal frames which are used to hold up outgoing lines from the main transformer, which impacts the noise reduction effect. In recent years, many scholars have paid attention to the utilization of combing active sound attenuation technology and sound barriers to reduce noises with a wide range of frequencies. If active sound barriers are used in these places, the noise diffraction of sound barriers can be effectively reduced ${ }^{[5]}$. This paper has explored a type of vertical active sound barrier to avoid

${ }^{\mathrm{a}}$ Sheng Hu: hbhusheng@163.com the interference of the outgoing lines of transformers, so as to reinforce the low-frequency noise attenuation effect of sound barriers.

\section{Design of the active sound barrier system}

Control objectives of the controller are closely related to the properties of noise sources, which are mainly spectral characteristic and propagation characteristic. The control system of the project adopts a distributed type of feed-forward controlled active control system, the structure diagram of which is shown in Fig. 1 and Fig. 2, including passive sound barrier 2 and distributed type of feed-forward controller array 4 . The cascade system is adopted to connect controllers in order to control the extension of the system to a multiple secondary path. Each active controller consists of reference sensor 5, error sensor 6, digital signal processing module 7 and secondary loudspeaker 8 . Moreover, a horizontal support 3 is installed at the top of the passive barrier with the height of $h_{b}$, and there is a equal spacing between controller units of the active controller array on the support, as shown in Figure 1; the distance between the centres of two control units is d, in order to achieve a better noise reduction effect, $d$ should be less than half of the corresponding wave length of the maximum control frequency ${ }^{[6]}$. When the primary sound source 1 radiates noises, reference sensor would collect the information of the primary sound field, and error sensor 6 would collect the information of the total sound field of the target controlling area, and the two signals

This is an Open Access article distributed under the terms of the Creative Commons Attribution License 4.0, which permits unrestricted use distribution, and reproduction in any medium, provided the original work is properly cited. 
would then be transferred to the digital signal processing module 7 , which adopts the single channel feed-forward adaptive algorithms, and the result is used to regulate the output of the secondary loudspeaker to minimize the amplitude square of sound pressure at the error sensor.

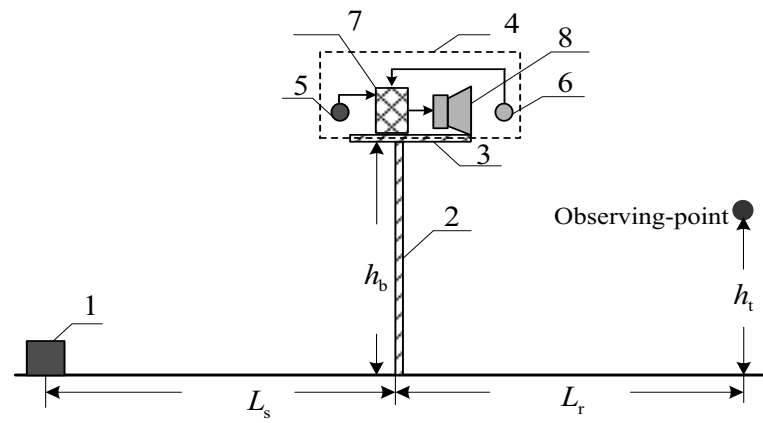

Figure 1. Structure diagram of the active sound barrier

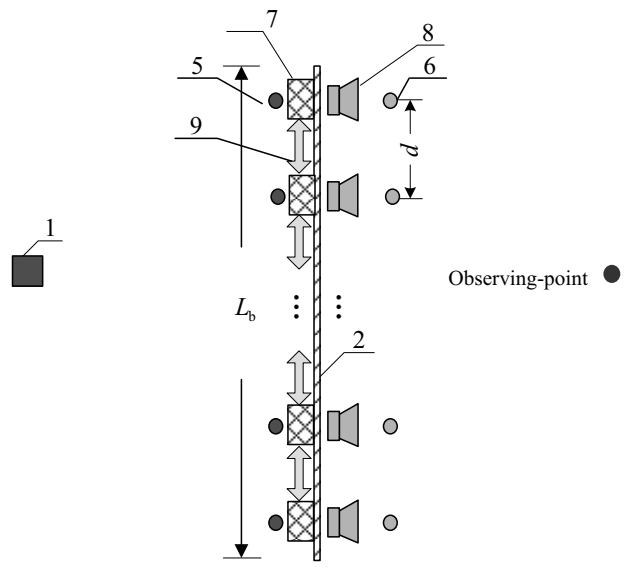

Figure 2. Structure diagram of the active sound barrier

\subsection{Hardware design of the controller}

The type of controller used in this project is a cascadable single-channel active controller, the schematic diagram of which is shown in Fig. 3. The quantity of the cascadable single-channel active controllers that form the controller can be changed as per the actual need. All the cascadable single-channel active controllers that form the controller are serially concatenated. The first unit is connected with the computer via a serial port communication module, and the computer sends out operating commands to any or all units via the computer's serial port, the serial port communication module of the first unit and the cascading communication module of each unit, or receives status information from any or all units. Thus the manipulation, commissioning and supervision of any unit can be performed in a more convenient and flexible way.

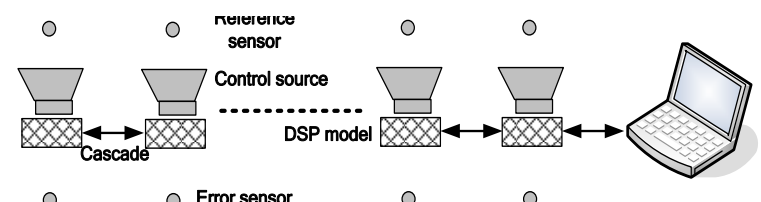

Figure 3. Structure diagram of the active sound barrier
The schematic diagram of an individual active sound controller is shown in Fig. 4. Reference sensors and error sensors would collect information from the noise source and the total sound field respectively, and then input them into the Digital Signal Processor (DSP) via a signal conditioner and A/D converter. The output signal from DSP would go through a D/A converter, filter circuit and power amplificatory circuit to drive the control source. The communication between DSP and the computer is conducted via the RS232 serial port (utilized in commissioning; only one unit uses the serial port in normal operation, and the communication of the other units is conducted via the cascading serial port). Moreover, system control can be realized by operating the interface of control software in the computer.

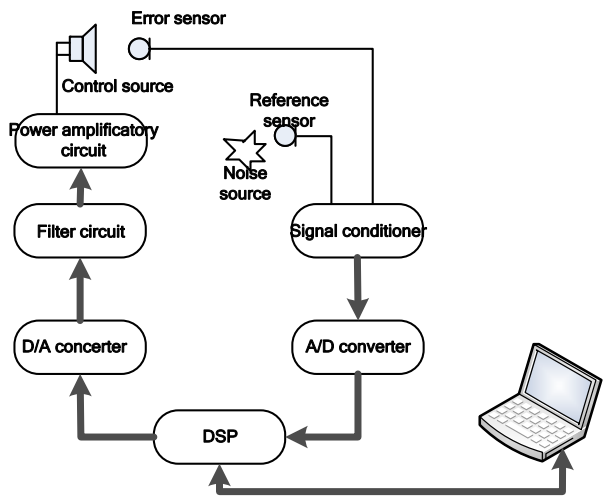

Figure 4. Structure diagram of the active sound barrier

\subsection{Layout of the active sound barriers}

In general, the software design of the controller can be divided into two parts, namely algorithm and auxiliary service. Algorithm, among which, is the core of the controller, which realizes the controller's basic functions, namely modeling and controlling, and auxiliary service includes operation maintenance, communication and User Interface (UI), etc. The software of this project is expected to be divided into five parts, as shown in Figure 5-9.(1) Feed-forward control algorithm software modules: include a modeling software module for the physical field between the secondary source and error sensors, a realtime signal control software module, and a filter updating control software module. These modules are performed on the real-time data processing chip contained in the DSP module of each unit.(2) Operation maintenance software modules: include an operation command processing software module and a status information reporting software module. These modules are performed on the real-time data processing chip contained in the DSP module of each unit.(3) Cascading communication software modules: performed on the real-time data processing chip contained in the DSP module of each unit.(4) Serial port communication software modules: include two parts; one part is performed on the real-time data processing chip contained in the DSP module of each unit, and the other is performed on the computer.(5) UI software modules: performed on the computer. The software modules on the data processing chip adopt the 
assembly language and $\mathrm{C}$ language programming, and the software modules on the computer adopt the LabWindows/CVI software programming by National Instruments (NI).

\section{Simulation result and analysis}

The effect of active noise reduction has been simulated by using a simple model. In the simulation process, suppose the noise source is mainly composed of sounds with mono-frequencies and the harmonic waves (such as $100 \mathrm{~Hz}, 200 \mathrm{~Hz}$ and $300 \mathrm{~Hz}$, which is a reasonable assumption for transformer noises), the acoustic centre of the noise source is 2 meters above the ground, and there is a sound barrier, with 3 meters in height and 8 meters in width, locating at 2 meters away from the acoustic centre. Moreover, 20 sets of single channel active noise control system are installed at the top of the active sound barrier (with the sensors and controlled sound source designed at the same level), and the extra insertion loss for noises with different frequencies can be obtained by numerical simulation, as shown in Fig. 5 to Fig. 8. It is thus evident that the extra insertion loss of the mono-frequencies ranges from approximately 5 to $15 \mathrm{~dB}$ in the acoustic shadow area, and has a slightly decrease in the other area, but still showing positive values; besides, there is extra insertion loss of 1 to $6 \mathrm{~dB}$ even at a height of 10 meters. In the meantime, the amount of noise reduction in the error sensors of each single channel control system should be higher than $12 \mathrm{~dB}$.

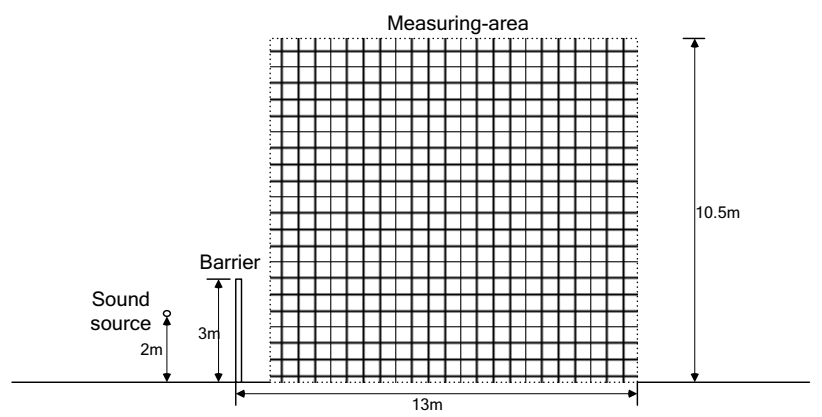

Figure 5. System location

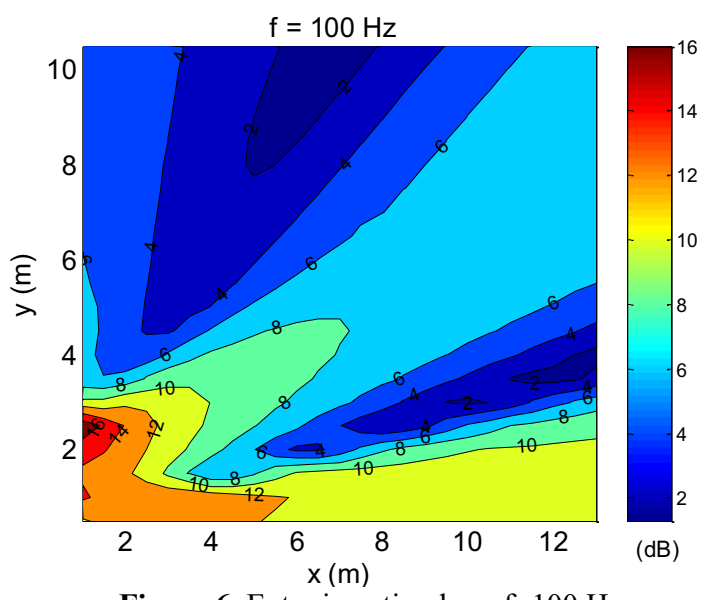

Figure 6. Extra insertion loss, $\mathrm{f}=100 \mathrm{~Hz}$

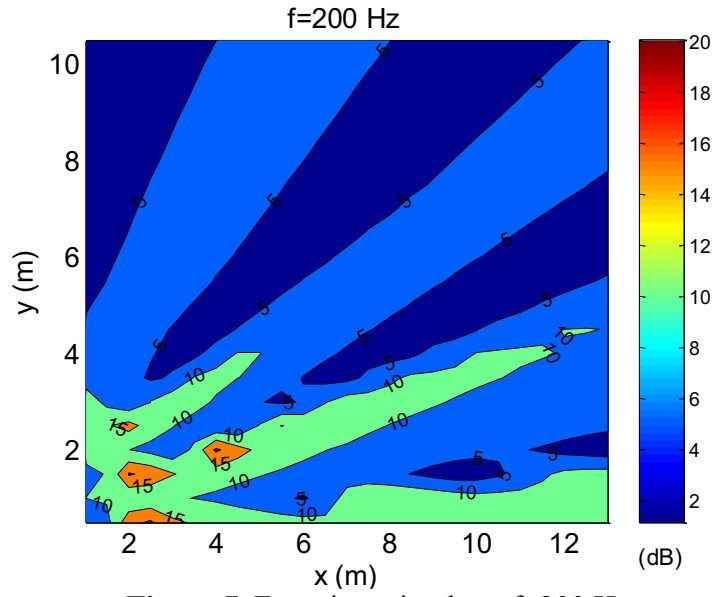

Figure 7. Extra insertion loss, $\mathrm{f}=200 \mathrm{~Hz}$

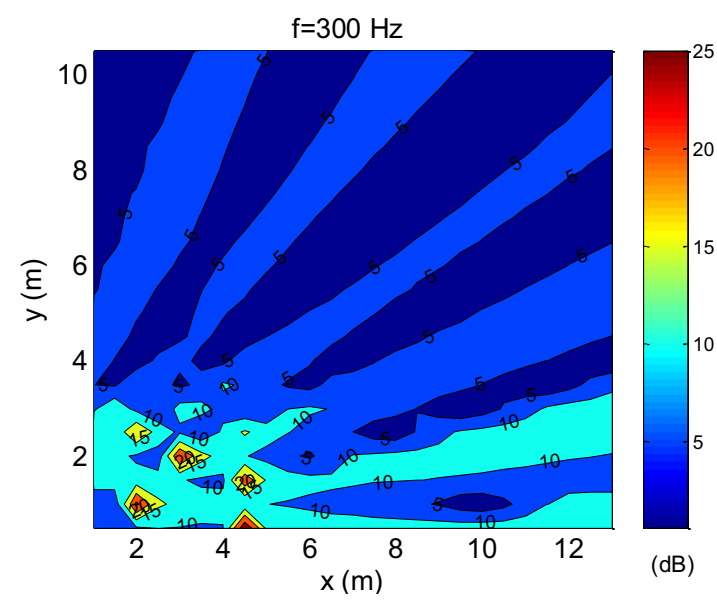

Figure 8. Extra insertion loss, $\mathrm{f}=300 \mathrm{~Hz}$

\section{On-site experiment}

\subsection{Layout of the active sound barriers}

The plan view of the substation is shown in Figure 6 . There are three main transformers equipped in the substation, with $110 \mathrm{KV}$ power distribution unit to the west, and reception office, $10 \mathrm{KV}$ high voltage room and control room surrounding the east. The substation itself is surrounded by residential areas, with its north bounding wall 2.0 meters away from a property management building, and east bounding wall 4.8 meters away from a 6-storey residential building and 7.0 meters away from a 16-storey residential building, and south bounding wall 32.0 meters away from a 14-storey residential building. In order to suppress the noise impact of the main transformers to their surrounding residential areas, a 12meter high sound barrier has been set up in three sides of the main transformers, as shown in the red parts of Figure 7. The barrier has a length of 16 meters in the north and south, and 51 meters in the east. For the convenience of maintaining transformers, 1-meter long and 6-meter wide barriers have been set up only at the two corners of the west of transformers, as shown in the blue parts of the figure.7, No.1 main transformer has the highest noise level, so the active noise control system is applied in the 
barrier abut No.1 main transformer in order to form an active sound barrier. Due to the restrictions of installation condition, active noise control system cannot be installed at the top of the existing barrier; therefore, a 15-channel active noise control system is set up vertically along the edge of the 1-meter long barrier in the southwest of No.1 main transformer, as shown in Fig. 9 and Fig. 10.
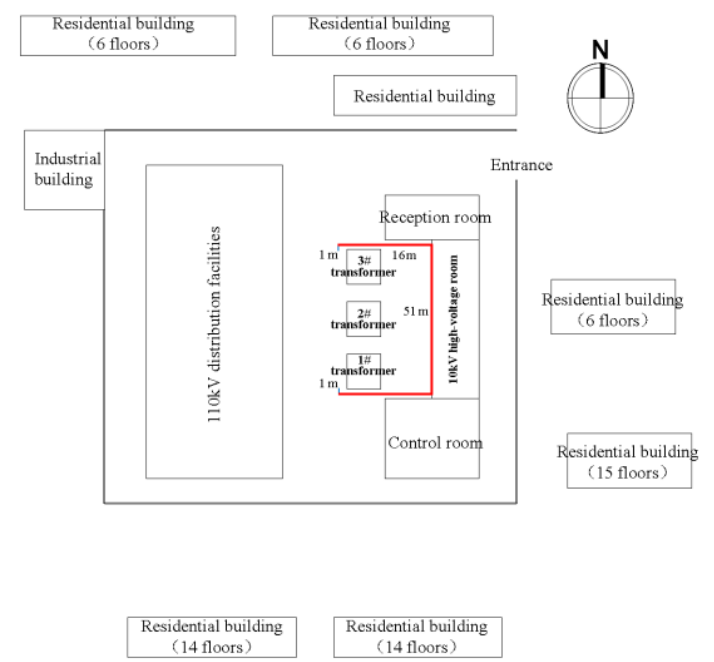

Figure 9. Plan layout of the substation

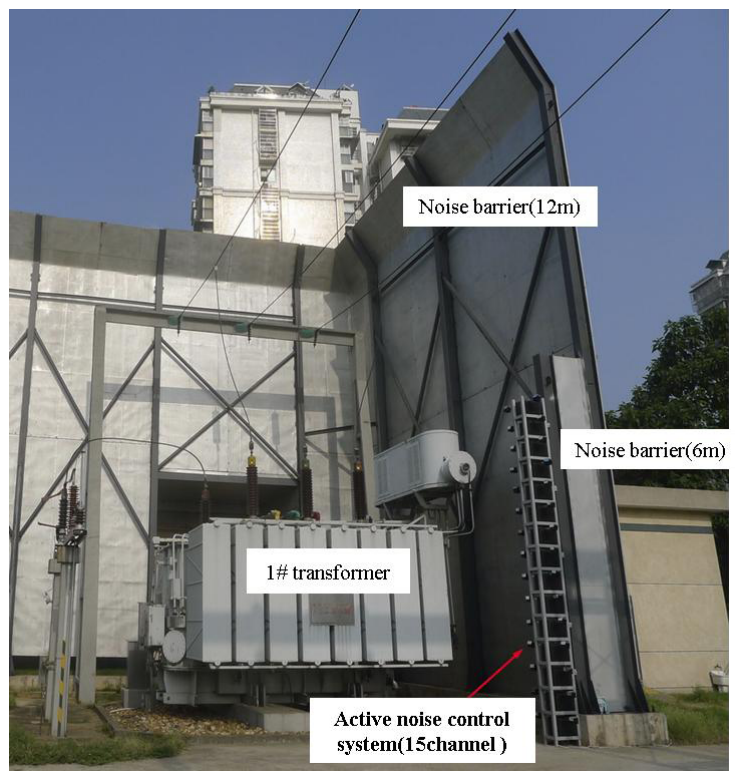

Figure 10. Picture of the active sound barriers at the substation

\subsection{Experimental result and analysis}

Seen from Table 1, active control has changed the sound field distribution in the space. The test result indicates that the active system has a certain effect in controlling noises with a frequency of less than $400 \mathrm{~Hz}$ at the factory boundary, and the decrease of A-weighted sound pressure level ranges from 0.3 to $4.3 \mathrm{~dB}$. The effect of the active noise control system is impacted by the layout of the active noise reduction system, the acoustic environment on site and the spectral characteristic of the target area.

Table 1. A-weighted sound pressure level of measuring points.

\begin{tabular}{|c|c|c|c|}
\hline $\begin{array}{c}\text { Measuring } \\
\text { Point No. }\end{array}$ & $\begin{array}{c}\text { active } \\
\text { control } \\
\text { on }\end{array}$ & $\begin{array}{c}\text { active } \\
\text { control } \\
\text { off }\end{array}$ & $\begin{array}{c}\text { Noise Reduction } \\
\text { Amount (dB(A)) }\end{array}$ \\
\hline 1 & 55.0 & 55.3 & -0.3 \\
\hline 2 & 55.7 & 53.6 & 2.1 \\
\hline 3 & 57.9 & 55.8 & 2.1 \\
\hline 4 & 57.2 & 56.7 & 0.5 \\
\hline 5 & 59.2 & 58.1 & 1.1 \\
\hline 6 & 58.0 & 54.6 & 3.4 \\
\hline $\begin{array}{l}\text { MNRV } \\
(\mathrm{dB}(\mathrm{A}))\end{array}$ & & & 1.5 \\
\hline
\end{tabular}

\section{Conclusions}

(1) According to the spectral characteristic and propagation characteristic of transformers, the essay has developed a distributed type of feed-forward controlled active sound barrier that can be applied to the noise control of transformers.(2) The simulation result of active sound barriers shows that the extra insertion loss of the mono-frequencies $(100 \mathrm{~Hz}, 200 \mathrm{~Hz}$ and $300 \mathrm{~Hz}$ to be specific) ranges from approximately 5 to $15 \mathrm{~dB}$ in the acoustic shadow area, and has a slightly decrease in the other area, but still showing positive values; besides, there is extra insertion loss of 1 to $6 \mathrm{~dB}$ even at a height of 10 meters.(3) An experiment using a 15-channel active sound barrier has been carried out in a $110 \mathrm{kV}$ substation. The result of the experiment shows that the MNRV are $1.5 \mathrm{~dB}$ and $1.8 \mathrm{~dB}(\mathrm{~A})$ respectively for the overall SPL and A-weighted SPL of the noise measuring points at the substation boundary. The effect of the active noise control system is impacted by the layout of the active noise control system, the acoustic environment on site and the spectral characteristic of the target area.

\section{References}

1. W. B. Conver, Noise Control, 2(1956)

2. N. Hesselmann, Appl. Acoust,11(1978)

3. O. L. Angevine, Pro. Inter. Noise 1992.

4. K.A. Chen, Active Noise Control(book), (2003)

5. H.S. Hai, X.F. Huang, S. Hu, X.J. Qiu, Pro. Inter. Noise 2014.

6. A. Omoto, K. Fujiwara, J. Acoust. Soc. Am., 94(1993) 